\title{
A FINITE ELEMENT ANALYSIS OF A PSEUDOSTRESS FORMULATION FOR THE STOKES EIGENVALUE PROBLEM
}

\author{
SALIM MEDDAHI, DAVID MORA, AND RODOLFO RODRÍGUEZ
}

\begin{abstract}
In this paper we analyze a finite element approximation of the Stokes eigenvalue problem. We present a variational formulation of the problem relying only on the pseudostress tensor. We present an $H$ (div)-conforming discretization of the problem by means of the lowest order Brezzi-DouglasMarini mixed finite element. We show that the resulting scheme provides a correct approximation of the spectrum and prove quasi-optimal error estimates. Finally, We present some numerical experiments supporting our theoretical results.
\end{abstract}

\section{INTRODUCTION}

The finite element approximation of eigenvalue problems is the object of great interest from both the practical and theoretical point of view. We refer to $[1,3$, $19]$ and the references therein for the state of art in this subject area. We are particularly interested here in the finite element analysis of the Stokes eigenvalue problem. The practical interest in Stokes eigenvalues and eigenmodes is explained in [16]. One motivation may be, for example, the study of a plate buckling problem. Indeed, it is well known that when this problem is subject to clamped boundary conditions it admits an equivalent formulation in terms of a Stokes problem, see $[19$, Section $7(d)]$ and $[7]$.

Two formulations of the Stokes eigenvalue problem were analyzed in Mercier et al. [19, Section $7(\mathrm{~d}, \mathrm{e})]$. More recently, an alternative study was presented in [4] (see also Part 3 of [3] and the references therein). This approach relies on the usual velocity-pressure formulation of the Stokes system and it was also used in [17] to perform an a posteriori error analysis. The aim of this paper is to propose a new formulation of the problem. We follow the strategy used in $[6,12,13]$ for the stationary source Stokes problem and introduce the so-called pseudostress tensor as a variable. Actually we will eliminate the pressure and velocity fields from the eigenvalue variational formulation and keep the pseudostress $\boldsymbol{\sigma}$ as the only unknown of the problem. As this variable will be sought in $\mathrm{H}(\operatorname{div} ; \Omega)$, we illustrate

2000 Mathematics Subject Classification. 65N25,76D07,65N30.

Key words and phrases. Stokes equations; eigenvalue problem, finite elements, error estimates.

The first author was partially supported by the Ministery of Education of Spain through the Project MTM2010-18427.

The second author was partially supported by CONICYT-Chile through FONDECYT project 11100180, by DIUBB through project 120808 GI/EF, and Anillo ANANUM, ACT1118, CONICYT (Chile).

The third author was partially supported by BASAL project CMM, Universidad de Chile (Chile) and Anillo ANANUM, ACT1118, CONICYT (Chile). 
our spectral approximation theory by means of the lowest order Brezzi-DouglasMarini (BDM) mixed finite element. It is worth mentioning that although we are using the pseudostress as a unique variable, the velocity and the pressure fields can be easily post-processed.

The well-known Babuška-Osborn abstract spectral approximation theory (see [1]) can not be used to deal with the analysis of our problem. Indeed, the kernel of the bilinear form defining the variational formulation has, in our case, an infinite dimensional kernel. The corresponding the solution operator is not compact and a nontrivial essential spectrum may, in such cases, originate a spectral pollution at the discrete level. We follow here [18], and take advantage of the classical theory developed in $[8,9]$ for non-compact operators, to prove that our numerical scheme provides a safe approximation of the eigenvalues at a quasi-optimal convergence rate.

The outline of this article is as follows: we introduce in section 2 the variational formulation of the eigenvalue Stokes problem and define the solution operator. Section 3 is devoted to the spectrum characterization of the solution operator. In section 4 , we introduce the discrete eigenvalue problem and describe the spectrum of the discrete solution operator. In section 5 , we prove that the numerical scheme provides a correct spectral approximation and establish quasi-optimal error estimates for the eigenvalues and eigenfunctions. Finally, we present in section 6 a set of numerical experiments to confirm that the method is not polluted with spurious modes and to show that the experimental rates of convergence are in accordance with the theoretical ones.

We end this section with some notations that will be used below. Given any Hilbert space $V$, let $V^{n}$ and $V^{n \times n}$ denote, respectively, the space of vectors and tensors of order $n(n=2$ or 3$)$ with entries in $V$. Given $\boldsymbol{\tau}:=\left(\tau_{i j}\right)$ and $\boldsymbol{\sigma}:=$ $\left(\sigma_{i j}\right) \in \mathbb{R}^{n \times n}$, we define as usual the transpose tensor $\boldsymbol{\tau}^{\mathrm{t}}:=\left(\tau_{j i}\right)$, the tensor inner product $\boldsymbol{\tau}: \boldsymbol{\sigma}:=\sum_{i, j=1}^{n} \tau_{i j} \sigma_{i j}$, the trace $\operatorname{tr} \boldsymbol{\tau}:=\sum_{i=1}^{n} \tau_{i i}$ and the deviatoric tensor $\boldsymbol{\tau}^{\mathrm{D}}:=\boldsymbol{\tau}-\frac{1}{n}(\operatorname{tr} \boldsymbol{\tau}) \boldsymbol{I}$, where $\boldsymbol{I}$ stands for the identity matrix of $\mathbb{R}^{n \times n}$.

Let $\Omega$ be a generic Lipschitz bounded domain of $\mathbb{R}^{n}$ with boundary $\partial \Omega$. For $s \geq 0$, $\|\cdot\|_{s, \Omega}$ stands indistinctly for the norm of the Hilbertian Sobolev spaces $\mathrm{H}^{s}(\Omega)$, $\mathrm{H}^{s}(\Omega)^{n}$ or $\mathrm{H}^{s}(\Omega)^{n \times n}$, with the convention $\mathrm{H}^{0}(\Omega):=\mathrm{L}^{2}(\Omega)$. We also define for $s \geq 0$ the Hilbert space $\mathrm{H}^{s}(\operatorname{div} ; \Omega):=\left\{\boldsymbol{\tau} \in \mathrm{H}^{s}(\Omega)^{n \times n}: \operatorname{div} \boldsymbol{\tau} \in \mathrm{H}^{s}(\Omega)^{n}\right\}$, whose norm is given by $\|\boldsymbol{\tau}\|_{\mathrm{H}^{s}(\operatorname{div} ; \Omega)}^{2}:=\|\boldsymbol{\tau}\|_{s, \Omega}^{2}+\|\operatorname{div} \boldsymbol{\tau}\|_{s, \Omega}^{2}$ and denote $\mathrm{H}(\operatorname{div} ; \Omega):=\mathrm{H}^{0}(\operatorname{div} ; \Omega)$.

Finally, we employ $\mathbf{0}$ to denote a generic null vector or tensor and use $C$ to denote generic constants independent of the discretization parameters, which may take different values at different places.

\section{The SPeCtral PRoblem}

Let $\Omega \subset \mathbb{R}^{n}(n=2,3)$ be a bounded and connected Lipschitz domain. We assume that the boundary $\partial \Omega$ admits a disjoint partition $\partial \Omega=\Gamma \cup \Sigma$ and denote by $\boldsymbol{\nu}$ the outward unit normal vector to $\partial \Omega$. 
The Stokes eigenvalue problem is formulated as follows (see [17]): Find $(\lambda, \boldsymbol{u}, p)$ such that,

$$
\left\{\begin{aligned}
-\operatorname{div}(\nabla \boldsymbol{u})+\nabla p & =\lambda \boldsymbol{u} & & \text { in } \Omega, \\
\operatorname{div} \boldsymbol{u} & =0 & & \text { in } \Omega, \\
\boldsymbol{u} & =0 & & \text { on } \Gamma, \\
(\nabla \boldsymbol{u}-p \boldsymbol{I}) \boldsymbol{\nu} & = & & \text { on } \Sigma .
\end{aligned}\right.
$$

Our aim is to employ a dual-mixed approach to derive a variational formulation of this problem. To this end, we introduce the pseudostress tensor (see [6])

$$
\boldsymbol{\sigma}:=\nabla \boldsymbol{u}-p \boldsymbol{I}
$$

and reformulate $(2.1)$ as follows in terms of this variable: Find $(\lambda, \boldsymbol{\sigma}, \boldsymbol{u})$ such that,

$$
\left\{\begin{array}{rlll}
-\operatorname{div} \boldsymbol{\sigma} & =\lambda \boldsymbol{u} & & \text { in } \Omega, \\
\boldsymbol{\sigma}^{\mathrm{D}}-\nabla \boldsymbol{u} & =0 & & \text { in } \Omega, \\
\boldsymbol{u} & =0 & & \text { on } \Gamma, \\
\boldsymbol{\sigma} \boldsymbol{\nu} & =0 & & \text { on } \Sigma .
\end{array}\right.
$$

We point out that the pressure $p$ disappeared from the formulation but it can be recovered since

$$
p=-\frac{1}{n} \operatorname{tr}(\boldsymbol{\sigma}) .
$$

We notice that the vector space

$$
\mathcal{W}:=\{\boldsymbol{\tau} \in \mathrm{H}(\operatorname{div} ; \Omega): \boldsymbol{\tau} \boldsymbol{\nu}=\mathbf{0} \text { on } \Sigma\}
$$

endowed with the $\mathrm{H}(\mathbf{d i v} ; \Omega)$-inner product is a Hilbert space. Testing the second equation of (2.3) with $\boldsymbol{\tau} \in \mathcal{W}$ and integrating by parts yield

$$
\int_{\Omega} \boldsymbol{\sigma}^{\mathrm{D}}: \boldsymbol{\tau}+\int_{\Omega} \boldsymbol{u} \cdot \operatorname{div} \boldsymbol{\tau}=0 .
$$

Next, we eliminate $\boldsymbol{u}$ from the last identity by using the first equation of (2.3) to obtain

$$
\int_{\Omega} \operatorname{div} \boldsymbol{\sigma} \cdot \operatorname{div} \boldsymbol{\tau}=\lambda \int_{\Omega} \boldsymbol{\sigma}^{\mathrm{D}}: \boldsymbol{\tau}^{\mathrm{D}} \quad \forall \boldsymbol{\tau} \in \mathcal{W},
$$

Consequently, the pseudostress Stokes eigenvalue formulation reads as follows:

Problem 1. Find $\lambda \in \mathbb{R}, 0 \neq \boldsymbol{\sigma} \in \mathcal{W}$, such that

$$
\int_{\Omega} \operatorname{div} \boldsymbol{\sigma} \cdot \operatorname{div} \boldsymbol{\tau}=\lambda \int_{\Omega} \sigma^{\mathrm{D}}: \boldsymbol{\tau}^{\mathrm{D}} \quad \forall \boldsymbol{\tau} \in \mathcal{W} .
$$

It is convenient to use a shift argument to rewrite this eigenvalue problem in the form:

Problem 2. Find $\lambda \in \mathbb{R}, 0 \neq \boldsymbol{\sigma} \in \mathcal{W}$, such that

$$
a(\boldsymbol{\sigma}, \boldsymbol{\tau})=(\lambda+1) b(\boldsymbol{\sigma}, \boldsymbol{\tau}) \quad \forall \boldsymbol{\tau} \in \mathcal{W},
$$

where the bounded bilinear forms $a(\cdot, \cdot)$ and $b(\cdot, \cdot)$ are given by

$$
\begin{aligned}
& a(\sigma, \tau):=\int_{\Omega} \sigma^{\mathrm{D}}: \tau^{\mathrm{D}}+\int_{\Omega} \operatorname{div} \boldsymbol{\sigma} \cdot \operatorname{div} \boldsymbol{\tau}, \quad \sigma, \boldsymbol{\tau} \in \mathcal{W}, \\
& b(\boldsymbol{\sigma}, \boldsymbol{\tau}):=\int_{\Omega} \boldsymbol{\sigma}^{\mathrm{D}}: \boldsymbol{\tau}^{\mathrm{D}}, \quad \boldsymbol{\sigma}, \boldsymbol{\tau} \in \mathcal{W} .
\end{aligned}
$$


We introduce the solution operator

$$
\begin{aligned}
T: \mathcal{W} & \longrightarrow \mathcal{W}, \\
f & \longmapsto T(f):=\sigma^{*},
\end{aligned}
$$

where $\sigma^{*} \in \mathcal{W}$ is the solution of the source problem

$$
a\left(\boldsymbol{\sigma}^{*}, \boldsymbol{\tau}\right)=b(\boldsymbol{f}, \boldsymbol{\tau}) \quad \forall \boldsymbol{\tau} \in \mathcal{W} .
$$

The following lemma allows us to establish the well-posedness of problem (2.5).

Lemma 2.1. There exists a constant $\alpha>0$, depending on $\Omega$, such that

$$
a(\boldsymbol{\tau}, \boldsymbol{\tau}) \geq \alpha\|\boldsymbol{\tau}\|_{\mathrm{H}(\operatorname{div} ; \Omega)} \quad \forall \boldsymbol{\tau} \in \mathcal{W} .
$$

Proof. For any $\boldsymbol{\tau} \in \mathrm{H}(\operatorname{div} ; \Omega)$, let $\boldsymbol{\tau}_{0}:=\boldsymbol{\tau}-\frac{1}{n|\Omega|}\left(\int_{\Omega} \operatorname{tr} \boldsymbol{\tau}\right) \boldsymbol{I}$. It is proved in $[5$, Proposition IV.3.1] that there exists $C_{1}>0$, depending only on $\Omega$, such that

$$
\left(\left\|\boldsymbol{\tau}^{\mathrm{D}}\right\|_{0, \Omega}^{2}+\|\operatorname{div} \boldsymbol{\tau}\|_{0, \Omega}^{2}\right) \geq C_{1}\left\|\boldsymbol{\tau}_{0}\right\|_{0, \Omega}^{2} \quad \forall \boldsymbol{\tau} \in \mathrm{H}(\operatorname{div} ; \Omega)
$$

On the other hand, one can easily adapt the proof from [11, Lemma 2.2] to show that there exists $C_{2}>0$, depending only on $\Omega$, such that

$$
\left\|\boldsymbol{\tau}_{0}\right\|_{\mathrm{H}(\mathrm{div} ; \Omega)}^{2} \geq C_{2}\|\boldsymbol{\tau}\|_{\mathrm{H}(\operatorname{div} ; \Omega)}^{2} \quad \forall \boldsymbol{\tau} \in \mathcal{W} .
$$

The result follows now immediately from the last two inequalities and the fact that $\operatorname{div} \tau_{0}=\operatorname{div} \tau$ in $\Omega$.

We deduce from Lemma 2.1 that the linear operator $\boldsymbol{T}$ is well defined and bounded. Notice that $(\lambda, \boldsymbol{\sigma}) \in \mathbb{R} \times \mathcal{W}$ solves Problem 1 if and only if $(1 /(1+\lambda), \boldsymbol{\sigma})$ is an eigenpair of $\boldsymbol{T}$, i.e., if and only if

$$
\boldsymbol{T} \boldsymbol{\sigma}=\mu \boldsymbol{\sigma}, \quad \text { with } \mu:=\frac{1}{1+\lambda} .
$$

It is important to realize that $\boldsymbol{T}$ is self-adjoint with respect to the inner product $a(\cdot, \cdot)$ in $\mathcal{W}$. Indeed, given $\boldsymbol{f}, \boldsymbol{g} \in \mathcal{W}$, we let $\boldsymbol{\sigma}$ and $\boldsymbol{\tau}$ be the solutions of (2.5) with sources $\boldsymbol{f}$ and $g$, respectively. Therefore $\sigma=\boldsymbol{T} \boldsymbol{f}$ and $\boldsymbol{\tau}=\boldsymbol{T} \boldsymbol{g}$ and

$$
a(\boldsymbol{T} \boldsymbol{f}, \boldsymbol{g})=a(\boldsymbol{\sigma}, \boldsymbol{g})=b(\boldsymbol{f}, \boldsymbol{g})=b(\boldsymbol{g}, \boldsymbol{f})=a(\boldsymbol{f}, \boldsymbol{\tau})=a(\boldsymbol{f}, \boldsymbol{T} \boldsymbol{g}) .
$$

\section{Spectral characterization}

Our aim here is to describe the spectrum $\operatorname{sp}(\boldsymbol{T})$ of the solution operator. To this end, we consider

$$
\mathcal{K}:=\{\boldsymbol{\tau} \in \mathcal{W}: \operatorname{div} \boldsymbol{\tau}=\mathbf{0} \text { in } \Omega\} .
$$

It is straightforward that $\left.\boldsymbol{T}\right|_{\mathcal{K}}: \mathcal{K} \longrightarrow \mathcal{K}$ reduces to the identity. Thus, $\mu=1$ is an eigenvalue of $\boldsymbol{T}$ and the corresponding eigenfunction $\boldsymbol{\sigma}$ satisfies by definition

$$
\int_{\Omega} \operatorname{div} \sigma \cdot \operatorname{div} \tau=0, \quad \forall \boldsymbol{\tau} \in \mathcal{W} .
$$

Consequently, the following result holds true.

Lemma 3.1. The operator $\boldsymbol{T}$ admits the eigenvalue $\mu=1$ and $\mathcal{K}$ is the associated eigenspace. 
Let us introduce now the auxiliary operator

$$
\begin{aligned}
P: \mathcal{W} & \longrightarrow \mathcal{W}, \\
\boldsymbol{\sigma} & \longmapsto P(\sigma):=\tilde{\sigma},
\end{aligned}
$$

where $(\widetilde{\boldsymbol{\sigma}}, \widetilde{\boldsymbol{u}}) \in \mathcal{W} \times \mathrm{L}^{2}(\Omega)^{n}$ is the solution of the following mixed problem:

$$
\begin{array}{lc}
\int_{\Omega} \tilde{\boldsymbol{\sigma}}^{\mathrm{D}}: \boldsymbol{\tau}^{\mathrm{D}}+\int_{\Omega} \tilde{\boldsymbol{u}} \cdot \operatorname{div} \boldsymbol{\tau}=0 & \forall \boldsymbol{\tau} \in \mathcal{W} \\
\int_{\Omega} \boldsymbol{v} \cdot \operatorname{div} \tilde{\boldsymbol{\sigma}}=\int_{\Omega} \boldsymbol{v} \cdot \operatorname{div} \boldsymbol{\sigma} & \forall \boldsymbol{v} \in \mathrm{L}^{2}(\Omega)^{n} .
\end{array}
$$

The Babuška-Brezzi theory shows that this problem is well posed. Indeed, it is well-known that the inf-sup condition

$$
\sup _{\boldsymbol{\tau} \in \mathcal{W}} \frac{\int_{\Omega} \boldsymbol{v} \cdot \operatorname{div} \boldsymbol{\tau}}{\|\boldsymbol{\tau}\|_{\mathrm{H}(\operatorname{div} ; \Omega)}} \geq \beta\|\boldsymbol{v}\|_{0, \Omega} \quad \forall v \in \mathrm{L}^{2}(\Omega)^{n}
$$

holds true and Lemma 2.1 guaranties that the bilinear form $\int_{\Omega} \sigma^{\mathrm{D}}: \tau^{\mathrm{D}}$ is elliptic on $\mathcal{K}$. Therefore, the linear operator $\boldsymbol{P}$ is well defined and bounded. Actually, Problem (3.1)-(3.2) is none other than the dual-mixed formulation of the following Stokes problem with external body force $-\operatorname{div} \boldsymbol{\sigma}$ :

$$
\begin{aligned}
-\operatorname{div} \widetilde{\boldsymbol{\sigma}}=-\operatorname{div} \boldsymbol{\sigma} & \text { in } \Omega, \\
\widetilde{\boldsymbol{\sigma}}^{\mathrm{D}}=\nabla \widetilde{\boldsymbol{u}} & \text { in } \Omega, \\
\widetilde{\boldsymbol{\sigma}} \boldsymbol{\nu}=\mathbf{0} & \text { on } \Sigma, \\
\widetilde{\boldsymbol{u}}=\mathbf{0} & \text { on } \Gamma .
\end{aligned}
$$

It is straightforward to check that $(\widetilde{\boldsymbol{\sigma}}, \widetilde{\boldsymbol{u}}) \in \mathrm{H}(\mathbf{d i v} ; \Omega) \times \mathrm{H}^{1}(\Omega)^{n}$ satisfies these equations if and only if $(\widetilde{\boldsymbol{\sigma}}, \widetilde{\boldsymbol{u}}) \in \mathcal{W} \times \mathrm{L}^{2}(\Omega)^{n}$ is the solution to (3.1)-(3.2).

Owing to the regularity result for the classical Stokes problem (see, for instance, $[10,14,21])$, we know that the solution $\widetilde{\boldsymbol{u}}$ to $(3.3)-(3.6)$ belongs to $\mathrm{H}^{1+s}(\Omega)^{n}$ for some $s \in(0,1]$ depending on the geometry of $\Omega$ and

$$
\|\widetilde{\boldsymbol{u}}\|_{1+s, \Omega} \leq C\|\operatorname{div} \boldsymbol{\sigma}\|_{0, \Omega},
$$

with $C>0$ independent of $\boldsymbol{\sigma}$. From now on, $s \in(0,1]$ denotes a constant such that this inequality holds true. As a consequence of this regularity result, we can state the following lemma.

Lemma 3.2. There exists $C>0$ such that for all $\boldsymbol{\sigma} \in \mathcal{W}$, if $(\tilde{\boldsymbol{\sigma}}, \tilde{\boldsymbol{u}}) \in \mathcal{W} \times \mathrm{L}^{2}(\Omega)^{n}$ is the solution to equations (3.1)-(3.2), then

$$
\|\widetilde{\boldsymbol{\sigma}}\|_{s, \Omega}+\|\widetilde{\boldsymbol{u}}\|_{1+s, \Omega} \leq C\|\operatorname{div} \boldsymbol{\sigma}\|_{0, \Omega}
$$

Consequently, $\boldsymbol{P}(\mathcal{W}) \subset \mathrm{H}^{s}(\Omega)^{n \times n}$.

It is easy to check that the operator $\boldsymbol{P}$ is idempotent and that its kernel is given by $\operatorname{Ker}(\boldsymbol{P})=\mathcal{K}$. Therefore, being $\boldsymbol{P}$ a projector, we have the direct sum $\mathcal{W}=\mathcal{K} \oplus \boldsymbol{P}(\mathcal{W})$. Moreover, it is clear that $\mathcal{K}$ and $\boldsymbol{P}(\mathcal{W})$ are orthogonal with respect to the inner product $a(\cdot, \cdot)$ of $\mathcal{W}$.

Lemma 3.3. The subspace $\boldsymbol{P}(\mathcal{W})$ is invariant for $\boldsymbol{T}$. 
Proof. The result is a direct consequence of the $a(\cdot, \cdot)$-orthogonality of the decomposition $\mathcal{W}=\mathcal{K} \oplus \boldsymbol{P}(\mathcal{W})$ and of the fact that $\boldsymbol{T}$ is self-adjoint operator with respect to the same bilinear form. Indeed, for any $\sigma \in \mathcal{W}$,

$$
a(\boldsymbol{T}(\boldsymbol{P} \boldsymbol{\sigma}), \boldsymbol{\tau}))=a(\boldsymbol{P} \boldsymbol{\sigma}, \boldsymbol{T} \boldsymbol{\tau})=a(\boldsymbol{P} \boldsymbol{\sigma}, \boldsymbol{\tau})=0 \quad \forall \boldsymbol{\tau} \in \mathcal{K} .
$$

Hence, $\boldsymbol{T}(\boldsymbol{P} \boldsymbol{\sigma})$ is orthogonal to $\mathcal{K}$ with respect to $a(\cdot, \cdot)$, which is equivalent to $\boldsymbol{T}(\boldsymbol{P} \boldsymbol{\sigma}) \in \boldsymbol{P}(\mathcal{W})$.

The properties of $\boldsymbol{T}$, as an operator from $\boldsymbol{P}(\boldsymbol{W})$ into itself, are established in the following result.

Proposition 3.1. The self-adjoint operator $\boldsymbol{T}$ satisfies

$$
\boldsymbol{T}(\boldsymbol{P}(\mathcal{W})) \subset\left\{\boldsymbol{\sigma}^{*} \in \mathrm{H}^{s}(\Omega)^{n \times n}: \operatorname{div} \boldsymbol{\sigma}^{*} \in \mathrm{H}^{1}(\Omega)^{n}\right\},
$$

and there exists $C>0$ such that for all $\boldsymbol{f} \in \boldsymbol{P}(\boldsymbol{W})$, if $\boldsymbol{\sigma}^{*}=\boldsymbol{T} \boldsymbol{f}$, then

$$
\left\|\boldsymbol{\sigma}^{*}\right\|_{s, \Omega}+\left\|\operatorname{div} \boldsymbol{\sigma}^{*}\right\|_{1, \Omega} \leq C\|\boldsymbol{f}\|_{\mathrm{H}(\operatorname{div} ; \Omega)} .
$$

Consequently, the operator $\left.\boldsymbol{T}\right|_{\boldsymbol{P}(\mathcal{W})}: \boldsymbol{P}(\mathcal{W}) \longrightarrow \boldsymbol{P}(\mathcal{W})$ is compact.

Proof. According to Lemma 3.3, $\left.\boldsymbol{T}\right|_{\boldsymbol{P}(\mathcal{W})}: \boldsymbol{P}(\mathcal{W}) \longrightarrow \boldsymbol{P}(\mathcal{W})$ is correctly defined. Let us consider $\boldsymbol{\sigma}^{*}=\boldsymbol{T} \boldsymbol{f}$ with $\boldsymbol{f} \in \boldsymbol{P}(\boldsymbol{W})$. Testing $(2.5)$ with $\boldsymbol{\tau} \in \mathcal{D}(\Omega)^{n \times n} \subset \mathcal{W}$ yields

$$
\boldsymbol{\sigma}^{* \mathrm{D}}-\nabla\left(\operatorname{div} \boldsymbol{\sigma}^{*}\right)=\boldsymbol{f}^{\mathrm{D}}
$$

which proves that $\operatorname{div} \boldsymbol{\sigma}^{*} \in \mathrm{H}^{1}(\Omega)^{n}$.

On the other hand, from Lemmas 3.3 and $3.2, \boldsymbol{\sigma}^{*} \in \boldsymbol{T}(\boldsymbol{P}(\mathcal{W})) \subset \boldsymbol{P}(\mathcal{W}) \subset$ $\mathrm{H}^{s}(\Omega)^{n \times n}$, so that (3.8) holds true and estimate (3.9) follows from Lemma 3.2. Finally, the compactness of $\left.\boldsymbol{T}\right|_{\boldsymbol{P}(\mathcal{W})}$ is a consequence of the compact embedding of the subspace

$$
\left\{\boldsymbol{\sigma}^{*} \in \mathrm{H}^{s}(\Omega)^{n \times n}: \operatorname{div} \boldsymbol{\sigma}^{*} \in \mathrm{H}^{1}(\Omega)^{n}\right\} \cap \mathcal{W}
$$

in $\mathcal{W}$.

We are now in a position to provide a spectral characterization of $\boldsymbol{T}$.

Theorem 3.1. The spectrum of $\boldsymbol{T}$ decomposes as follows: $\operatorname{sp}(\boldsymbol{T})=\{0,1\} \cup\left\{\mu_{k}\right\}_{k \in \mathbb{N}}$, where:

i) $\mu=1$ is an infinite-multiplicity eigenvalue of $\boldsymbol{T}$ and its associated eigenspace is $\mathcal{K}$;

ii) $\left\{\mu_{k}\right\}_{k \in \mathbb{N}} \subset(0,1)$ is a sequence of finite-multiplicity eigenvalues of $\boldsymbol{T}$ which converge to 0 and the corresponding eigenspaces lie in $\boldsymbol{P}(\mathcal{W}) ;$ moreover, the ascent of each of these eigenvalues is 1 ;

iii) $\mu=0$ is an eigenvalue of $\boldsymbol{T}$ and its associated eigenspace is $\left\{\boldsymbol{\tau} \in \mathcal{W}: \boldsymbol{\tau}^{\mathrm{D}}=\right.$ $0\}=\left\{q \boldsymbol{I}: q \in \mathrm{H}^{1}(\Omega)\right.$, and $q=0$ on $\left.\Sigma\right\}$.

Proof. As $\mathcal{W}=\mathcal{K} \oplus \boldsymbol{P}(\mathcal{W}),\left.\boldsymbol{T}\right|_{\mathcal{K}}: \mathcal{K} \longrightarrow \mathcal{K}$ reduces to the identity and $\left.\boldsymbol{T}\right|_{\boldsymbol{P}(\mathcal{W})}$ : $\boldsymbol{P}(\mathcal{W}) \longrightarrow \boldsymbol{P}(\mathcal{W})$ is compact (cf. Proposition 3.1), the decomposition of $\operatorname{sp}(\boldsymbol{T})$ follows immediately from the classical spectral characterization of compact operators. Property (i) was established in Lemma 3.1. Property (ii) follows from Proposition 3.1. Finally, it is easy to check that $\left\{\boldsymbol{\tau} \in \mathcal{W}: \boldsymbol{\tau}^{\mathrm{D}}=0\right\}$ is the eigenspace of $\boldsymbol{T}$ associated to $\mu=0$. The result follows by noting that $\boldsymbol{\tau}^{\mathrm{D}}=0$ if and only if $\boldsymbol{\tau}=q \boldsymbol{I}$, with $q=\frac{1}{n} \operatorname{tr} \boldsymbol{\tau} \in \mathrm{L}^{2}(\Omega), \nabla q=\operatorname{div} \boldsymbol{\tau} \in \mathrm{L}^{2}(\Omega)^{n}$ and $q \boldsymbol{n}=\boldsymbol{\tau} \boldsymbol{n}$ on $\Sigma$. 
As an immediate consequence of Proposition 3.1 (cf. (3.9)) we have the following additional regularity result for the eigenfunctions of $\boldsymbol{T}$ lying in $\boldsymbol{P}(\mathcal{W})$.

Corollary 3.1. Let $\boldsymbol{\sigma} \in \mathcal{W}$ be an eigenfunction of $\boldsymbol{T}$ associated to an eigenvalue $\mu \in(0,1)$. Then, $\boldsymbol{\sigma} \in \mathrm{H}^{s}(\Omega)^{n \times n}$, $\operatorname{div} \boldsymbol{\sigma} \in \mathrm{H}^{1}(\Omega)^{n}$, and

$$
\|\boldsymbol{\sigma}\|_{s, \Omega}+\|\operatorname{div} \boldsymbol{\sigma}\|_{1, \Omega} \leq C\|\boldsymbol{\sigma}\|_{\mathrm{H}(\operatorname{div} ; \Omega)},
$$

with $C>0$ depending on the eigenvalue.

\section{The Discrete PROBLEM}

Let $\left\{\mathcal{T}_{h}(\Omega)\right\}_{h>0}$ be a shape-regular family of triangulations of the polyhedral (polygonal) domain $\Omega$ by tetrahedra (triangles) $T$ with mesh size $h$. In what follows, given an integer $k \geq 0$ and a subset $S$ of $\mathbb{R}^{n}, \mathcal{P}_{k}(S)$ denotes the space of polynomials defined in $S$ of total degree less or equal than $k$.

We define the discrete version of $\mathcal{W}$ by

$$
\mathcal{W}_{h}:=\left\{\boldsymbol{\tau}_{h} \in \mathcal{W}:\left.\boldsymbol{\tau}_{h}\right|_{T} \in \mathcal{P}_{1}(T)^{n \times n} \forall T \in \mathcal{T}_{h}(\Omega)\right\}
$$

In addition, for the analysis below, we will need the space

$$
\mathcal{U}_{h}:=\left\{\boldsymbol{v}_{h} \in \mathrm{L}^{2}(\Omega)^{n}:\left.\boldsymbol{v}_{h}\right|_{T} \in \mathcal{P}_{0}(T)^{n} \forall T \in \mathcal{T}_{h}(\Omega)\right\} .
$$

Let us recall now some well-known approximation properties of the finite element spaces introduced above. Given $s \in(0,1]$, we denote by $\Pi_{h}: \mathrm{H}^{s}(\Omega)^{n \times n} \cap \mathcal{W} \rightarrow \mathcal{W}_{h}$ the usual BDM interpolation operator (see [5]) characterized by the conditions:

$$
\int_{F}\left(\boldsymbol{\Pi}_{h} \boldsymbol{\tau}\right) \boldsymbol{\nu}_{F} \cdot \boldsymbol{p}=\int_{F} \boldsymbol{\tau} \boldsymbol{\nu}_{F} \cdot \boldsymbol{p} \quad \forall \boldsymbol{p} \in \mathcal{P}_{1}(F)^{n}
$$

for all face (edge) $F$ of $T \in \mathcal{T}_{h}(\Omega)$, where $\boldsymbol{\nu}_{F}$ is a unit normal vector to the face (edge) $F$. The following commuting diagram property holds true (cf. [5]):

$$
\operatorname{div}\left(\boldsymbol{\Pi}_{h} \boldsymbol{\tau}\right)=\boldsymbol{L}_{h}(\operatorname{div} \boldsymbol{\tau}) \quad \forall \boldsymbol{\tau} \in \mathrm{H}^{s}(\Omega)^{n \times n} \cap \mathrm{H}(\operatorname{div} ; \Omega),
$$

where $\boldsymbol{L}_{h}: \mathrm{L}^{2}(\Omega)^{n} \longrightarrow \mathcal{U}_{h}$ is the $\mathrm{L}^{2}(\Omega)^{n}$-orthogonal projector. In addition, it is well-known (see, e.g., [15, Theorem 3.16]) that there exists $C>0$, independent of $h$, such that for any $\boldsymbol{\tau} \in \mathrm{H}^{s}(\Omega)^{n \times n} \cap \mathrm{H}(\operatorname{div} ; \Omega)$ there holds

$$
\left\|\boldsymbol{\tau}-\boldsymbol{\Pi}_{h} \boldsymbol{\tau}\right\|_{0, \Omega} \leq C h^{s}\left(\|\boldsymbol{\tau}\|_{s, \Omega}+\|\operatorname{div} \boldsymbol{\tau}\|_{0, \Omega}\right) .
$$

Thus, for any $s \in(0,1]$, we have:

$$
\begin{array}{rlrl}
\left\|\boldsymbol{v}-\boldsymbol{L}_{h} \boldsymbol{v}\right\|_{0, \Omega} & \leq C h^{s}\|\boldsymbol{v}\|_{s, \Omega} & & \forall \boldsymbol{v} \in \mathrm{H}^{s}(\Omega)^{n}, \\
\left\|\boldsymbol{\tau}-\boldsymbol{\Pi}_{h} \boldsymbol{\tau}\right\|_{\mathrm{H}(\operatorname{div} ; \Omega)} \leq C h^{s}\|\boldsymbol{\tau}\|_{\mathrm{H}^{s}(\operatorname{div} ; \Omega)} & & \forall \boldsymbol{\tau} \in \mathrm{H}^{s}(\operatorname{div} ; \Omega) \cap \mathcal{W} .
\end{array}
$$

Actually, (4.4) is a straightforward consequence of (4.1), (4.2), and (4.3).

Let us now introduce the discrete counterpart of Problem 1.

Problem 3. Find $\lambda_{h} \in \mathbb{R}, 0 \neq \sigma_{h} \in \mathcal{W}_{h}$, such that

$$
\int_{\Omega} \operatorname{div} \sigma_{h} \cdot \operatorname{div} \tau_{h}=\lambda_{h} \int_{\Omega} \sigma_{h}^{\mathrm{D}}: \tau_{h}^{\mathrm{D}} \quad \forall \boldsymbol{\tau}_{h} \in \mathcal{W}_{h} .
$$

The discrete version of the operator $\boldsymbol{T}$ is then given by

$$
\begin{aligned}
\widetilde{\boldsymbol{T}}_{h}: \mathcal{W} & \longrightarrow \mathcal{W}, \\
\boldsymbol{f} & \longmapsto \widetilde{\boldsymbol{T}}_{h}(\boldsymbol{f}):=\sigma_{h}^{*},
\end{aligned}
$$


where $\boldsymbol{\sigma}_{h}^{*} \in \mathcal{W}_{h}$ is the solution of the discrete source problem,

$$
a\left(\boldsymbol{\sigma}_{h}^{*}, \boldsymbol{\tau}_{h}\right)=b\left(\boldsymbol{f}, \boldsymbol{\tau}_{h}\right) \quad \forall \boldsymbol{\tau}_{h} \in \mathcal{W}_{h} .
$$

Because of Lemma 2.1 and the Lax-Milgram Theorem, $\widetilde{\boldsymbol{T}}_{h}$ is well defined and uniformly bounded with respect to $h$. Moreover, Céa lemma ensures the existence of a constant $C>0$, independent of $h$, such that for all $\sigma \in \mathcal{W}$,

$$
\left\|\boldsymbol{T} \boldsymbol{\sigma}-\widetilde{\boldsymbol{T}}_{h} \boldsymbol{\sigma}\right\|_{\mathrm{H}(\mathrm{div} ; \Omega)} \leq C \inf _{\boldsymbol{\tau}_{h} \in \mathcal{W}_{h}}\left\|\boldsymbol{T} \boldsymbol{\sigma}-\boldsymbol{\tau}_{h}\right\|_{\mathrm{H}(\mathrm{div} ; \Omega)} .
$$

Notice that, as $\widetilde{\boldsymbol{T}}_{h}(\mathcal{W}) \subset \mathcal{W}_{h}$, we are allowed to introduce

$$
\boldsymbol{T}_{h}:=\left.\widetilde{\boldsymbol{T}}_{h}\right|_{\mathcal{W}_{h}}: \mathcal{W}_{h} \longrightarrow \mathcal{W}_{h} .
$$

It is well-known that $\operatorname{sp}\left(\widetilde{\boldsymbol{T}}_{h}\right)=\operatorname{sp}\left(\boldsymbol{T}_{h}\right) \cup\{0\}$ (see, for instance, [2, Lemma 4.1]).

Once more, as in the continuous case, $\left(\lambda_{h}, \boldsymbol{\sigma}_{h}\right) \in \mathbb{R} \times \mathcal{W}_{h}$ solves Problem 3 if and only if $\left(1 /\left(1+\lambda_{h}\right), \boldsymbol{\sigma}_{h}\right)$ is an eigenpair of $\boldsymbol{T}_{h}$, i.e., if and only if

$$
\boldsymbol{T}_{h} \boldsymbol{\sigma}_{h}=\mu_{h} \boldsymbol{\sigma}_{h}, \quad \text { with } \mu_{h}:=\frac{1}{1+\lambda_{h}} .
$$

Moreover, $\boldsymbol{T}_{h}$ is also self-adjoint with respect to $a(\cdot, \cdot)$. To describe the spectrum of this operator, we will proceed as in the continuous case and decompose $\mathcal{W}_{h}$ into a convenient direct sum. To this end, we define

$$
\mathcal{K}_{h}:=\mathcal{K} \cap \mathcal{W}_{h}=\left\{\boldsymbol{\tau}_{h} \in \mathcal{W}_{h}: \operatorname{div} \boldsymbol{\tau}_{h}=\mathbf{0} \text { in } \Omega\right\}
$$

and notice that, here again, $\left.\boldsymbol{T}_{h}\right|_{\mathcal{K}_{h}}: \mathcal{K}_{h} \longrightarrow \mathcal{K}_{h}$ reduces to the identity. Hence, $\mu_{h}=1$ is an eigenvalue of $\boldsymbol{T}_{h}$ and $\mathcal{K}_{h}$ is the associated eigenspace. Therefore, we have the following discrete analogue to Lemma 3.1.

Lemma 4.1. The operator $\boldsymbol{T}_{h}$ admits the eigenvalue $\mu_{h}=1$ and $\mathcal{K}_{h}$ is the associated eigenspace.

We define the discrete version of the auxiliary operator $\boldsymbol{P}$ by

$$
\begin{aligned}
\boldsymbol{P}_{h}: \mathcal{W} & \longrightarrow \mathcal{W}_{h}, \\
\boldsymbol{\sigma} & \longmapsto \boldsymbol{P}_{h}(\boldsymbol{\sigma}):=\widetilde{\boldsymbol{\sigma}}_{h},
\end{aligned}
$$

where $\left(\widetilde{\boldsymbol{\sigma}}_{h}, \widetilde{\boldsymbol{u}}_{h}\right) \in \mathcal{W}_{h} \times \mathcal{U}_{h}$ is the solution of the following mixed discrete problem:

$$
\begin{array}{ll}
\int_{\Omega} \widetilde{\boldsymbol{\sigma}}_{h}^{\mathrm{D}}: \boldsymbol{\tau}_{h}^{\mathrm{D}}+\int_{\Omega} \widetilde{\boldsymbol{u}}_{h} \cdot \operatorname{div} \boldsymbol{\tau}_{h}=0 & \forall \boldsymbol{\tau}_{h} \in \mathcal{W}_{h}, \\
\int_{\Omega} \boldsymbol{v}_{h} \cdot \operatorname{div} \widetilde{\boldsymbol{\sigma}}_{h}=\int_{\Omega} \boldsymbol{v}_{h} \cdot \operatorname{div} \boldsymbol{\sigma} & \forall \boldsymbol{v}_{h} \in \mathcal{U}_{h} .
\end{array}
$$

We point out that the inf-sup condition

$$
\sup _{\boldsymbol{\tau}_{h} \in \mathcal{W}_{h}} \frac{\int_{\Omega} \boldsymbol{v}_{h} \cdot \operatorname{div} \boldsymbol{\tau}_{h}}{\left\|\boldsymbol{\tau}_{h}\right\|_{\mathrm{H}(\mathbf{d i v} ; \Omega)}} \geq \hat{\beta}\left\|\boldsymbol{v}_{h}\right\|_{0, \Omega} \quad \forall v_{h} \in \mathcal{U}_{h}
$$

is satisfied with a constant $\hat{\beta}$ independent of $h$ (see [5]). Moreover, the ellipticity of the bilinear form $\int_{\Omega} \sigma^{\mathrm{D}}: \boldsymbol{\tau}^{\mathrm{D}}$ on the discrete kernel $\mathcal{K}_{h}$ follows easily from Lemma 2.1 and the fact that $\operatorname{div}\left(\mathcal{W}_{h}\right) \subset \mathcal{U}_{h}$. Hence, as a consequence of the Babuška-Brezzi 
theory, problem (4.6)-(4.7) is well posed. The linear operator $\boldsymbol{P}_{h}$ is then well defined and uniformly bounded with respect to $h$. Finally, the following Céa estimate holds true:

$$
\begin{aligned}
\left\|\widetilde{\boldsymbol{\sigma}}-\widetilde{\boldsymbol{\sigma}}_{h}\right\|_{\mathrm{H}(\mathbf{d i v} ; \Omega)}+ & \left\|\widetilde{\boldsymbol{u}}-\widetilde{\boldsymbol{u}}_{h}\right\|_{0, \Omega} \\
& \leq C\left[\inf _{\boldsymbol{\tau}_{h} \in \mathcal{W}_{h}}\left\|\widetilde{\boldsymbol{\sigma}}-\boldsymbol{\tau}_{h}\right\|_{\mathrm{H}(\operatorname{div} ; \Omega)}+\inf _{\boldsymbol{v}_{h} \in \mathcal{U}_{h}}\left\|\widetilde{\boldsymbol{u}}-\boldsymbol{v}_{h}\right\|_{0, \Omega}\right],
\end{aligned}
$$

where $(\widetilde{\boldsymbol{\sigma}}, \widetilde{\boldsymbol{u}})$ and $\left(\widetilde{\boldsymbol{\sigma}}_{h}, \widetilde{\boldsymbol{u}}_{h}\right)$ are the solutions to (3.1)-(3.2) and (4.6)-(4.7), respectively. This estimate, combined with the approximation properties (4.4)-(4.3), leads to

$$
\left\|\boldsymbol{P} \boldsymbol{\sigma}-\boldsymbol{P}_{h} \boldsymbol{\sigma}\right\|_{\mathrm{H}(\operatorname{div} ; \Omega)} \leq C h^{s}\left[\|\widetilde{\boldsymbol{\sigma}}\|_{\mathrm{H}^{s}(\mathbf{d i v} ; \Omega)}+\|\widetilde{\boldsymbol{u}}\|_{s, \Omega}\right]
$$

whenever $\widetilde{\boldsymbol{\sigma}} \in \mathrm{H}^{s}(\Omega)^{n \times n}, \widetilde{\boldsymbol{u}} \in \mathrm{H}^{s}(\Omega)^{n}$, and $\operatorname{div} \widetilde{\boldsymbol{\sigma}} \in \mathrm{H}^{s}(\Omega)^{n}$. We already know from Lemma 3.2 that $\widetilde{\boldsymbol{\sigma}} \in \mathrm{H}^{s}(\Omega)^{n \times n}$ and $\widetilde{\boldsymbol{u}} \in \mathrm{H}^{s}(\Omega)^{n}$. However, $\operatorname{div} \widetilde{\boldsymbol{\sigma}}$ cannot be in $\mathrm{H}^{s}(\Omega)^{n}$ when $\boldsymbol{\sigma}$ is arbitrary in $\mathcal{W}$. Indeed, from (3.2), $\operatorname{div} \widetilde{\boldsymbol{\sigma}}=\operatorname{div} \boldsymbol{\sigma}$ which is in general only in $L^{2}(\Omega)^{n}$. In spite of this fact, there are two cases (see Lemma 4.2 below) in which an $\mathcal{O}\left(h^{s}\right)$ convergence for $\left\|\boldsymbol{P} \boldsymbol{\sigma}-\boldsymbol{P}_{h} \boldsymbol{\sigma}\right\|_{\mathrm{H}(\mathrm{div} ; \Omega)}$ can be proved. Fortunately, this is enough to develop the spectral approximation theory of our problem.

Lemma 4.2. There exists $C>0$ such that:

i) if $\boldsymbol{\sigma}$ is an eigenfunction of $\boldsymbol{T}$ associated to an eigenvalue $\mu \in(0,1)$, then

$$
\left\|\boldsymbol{P} \boldsymbol{\sigma}-\boldsymbol{P}_{h} \boldsymbol{\sigma}\right\|_{\mathrm{H}(\mathrm{div} ; \Omega)} \leq C h^{s}\|\boldsymbol{\sigma}\|_{\mathrm{H}(\mathrm{div} ; \Omega)} ;
$$

ii) if $\boldsymbol{\sigma}_{h} \in \mathcal{W}_{h}$, then

$$
\left\|\boldsymbol{P} \boldsymbol{\sigma}_{h}-\boldsymbol{P}_{h} \boldsymbol{\sigma}_{h}\right\|_{\mathrm{H}(\operatorname{div} ; \Omega)} \leq C h^{s}\left\|\operatorname{div} \boldsymbol{\sigma}_{h}\right\|_{0, \Omega} .
$$

Proof. Case (i). The estimate follows from (4.9), Lemma 3.2, and Corollary 3.1.

Case (ii). For $\boldsymbol{\sigma}_{h} \in \mathcal{W}_{h}$, let $\widetilde{\boldsymbol{\sigma}}=\boldsymbol{P} \boldsymbol{\sigma}_{h}$ and $\widetilde{\boldsymbol{\sigma}}_{h}=\boldsymbol{P}_{h} \boldsymbol{\sigma}_{h}$. By virtue of (4.8), (4.3) and Lemma 3.2, we have that

$$
\left\|\boldsymbol{P} \boldsymbol{\sigma}_{h}-\boldsymbol{P}_{h} \boldsymbol{\sigma}_{h}\right\|_{\mathrm{H}(\operatorname{div} ; \Omega)} \leq C\left[\inf _{\boldsymbol{\tau}_{h} \in \mathcal{W} \mathcal{W}_{h}}\left\|\widetilde{\boldsymbol{\sigma}}-\boldsymbol{\tau}_{h}\right\|_{\mathrm{H}(\operatorname{div} ; \Omega)}+h^{s}\left\|\operatorname{div} \boldsymbol{\sigma}_{h}\right\|_{0, \Omega}\right] .
$$

Now, as $\tilde{\boldsymbol{\sigma}} \in \mathcal{W} \cap \mathrm{H}^{s}(\Omega)^{n \times n}$ (cf. Lemma 3.2), $\boldsymbol{\tau}_{h}:=\boldsymbol{\Pi}_{h} \tilde{\boldsymbol{\sigma}} \in \mathcal{W}_{h}$ is well defined and, according to (4.2),

$$
\left\|\widetilde{\boldsymbol{\sigma}}-\boldsymbol{\Pi}_{h} \tilde{\boldsymbol{\sigma}}\right\|_{0, \Omega} \leq C h^{s}\left(\|\tilde{\boldsymbol{\sigma}}\|_{s, \Omega}+\|\operatorname{div} \tilde{\boldsymbol{\sigma}}\|_{0, \Omega}\right) .
$$

On the other hand, from (3.2), $\operatorname{div} \widetilde{\boldsymbol{\sigma}}=\boldsymbol{\operatorname { d i v }} \boldsymbol{\sigma}_{h}$ in $\Omega$. Therefore, because of (4.1),

$$
\operatorname{div}\left(\Pi_{h} \widetilde{\boldsymbol{\sigma}}\right)=\boldsymbol{L}_{h}(\operatorname{div} \widetilde{\boldsymbol{\sigma}})=\boldsymbol{L}_{h}\left(\operatorname{div} \boldsymbol{\sigma}_{h}\right)=\operatorname{div} \boldsymbol{\sigma}_{h}=\operatorname{div} \widetilde{\boldsymbol{\sigma}},
$$

which proves that

$$
\left\|\tilde{\boldsymbol{\sigma}}-\boldsymbol{\Pi}_{h} \tilde{\boldsymbol{\sigma}}\right\|_{\mathrm{H}(\operatorname{div} ; \Omega)}=\left\|\tilde{\boldsymbol{\sigma}}-\boldsymbol{\Pi}_{h} \tilde{\boldsymbol{\sigma}}\right\|_{0, \Omega}
$$

and the result follows.

We point out that for any $\boldsymbol{\sigma} \in \mathcal{W},(4.7)$ implies that

$$
\int_{\Omega} \boldsymbol{v}_{h} \cdot \operatorname{div}\left(\boldsymbol{P}_{h} \boldsymbol{\sigma}\right)=\int_{\Omega} \boldsymbol{v}_{h} \cdot \operatorname{div} \boldsymbol{\sigma}, \quad \forall \boldsymbol{v}_{h} \in \mathcal{U}_{h}
$$

Consequently, $\boldsymbol{P}_{h} \mid \mathcal{W}_{h}$ is idempotent which permits us to write $\mathcal{W}_{h}$ as a direct sum of the kernel $\mathcal{K}_{h}$ of $\left.\boldsymbol{P}_{h}\right|_{\mathcal{W}_{h}}$ and its range $\boldsymbol{P}_{h}\left(\mathcal{W}_{h}\right)$, i.e., $\mathcal{W}_{h}=\mathcal{K}_{h} \oplus \boldsymbol{P}_{h}\left(\mathcal{W}_{h}\right)$. 
Actually, it is easy to show that this decomposition is orthogonal with respect to $a(\cdot, \cdot)$. Consequently, the proof of Lemma 3.3 can be reproduced verbatim to obtain the following analogue result.

Lemma 4.3. The subspace $\boldsymbol{P}_{h}\left(\mathcal{W}_{h}\right)$ is invariant for $\boldsymbol{T}_{h}$.

Theorem 4.1. The spectrum of $\boldsymbol{T}_{h}$ consists of $M:=\operatorname{dim}\left(\mathcal{W}_{h}\right)$ eigenvalues, repeated accordingly to their respective multiplicities. The spectrum decomposes as follows: $\operatorname{sp}\left(\boldsymbol{T}_{h}\right)=\{0,1\} \cup\left\{\mu_{h k}\right\}_{k=1}^{K}$. Moreover,

i) the eigenspace associated with $\mu_{h}=1$ is $\mathcal{K}_{h}$;

ii) $\mu_{h k} \in(0,1), k=1, \ldots, K:=M-\operatorname{dim}\left(\mathcal{K}_{h}\right)$, are nondefective eigenvalues with eigenspaces lying on $\boldsymbol{P}_{h}\left(\mathcal{W}_{h}\right)$;

iii) $\mu_{h}=0$ is an eigenvalue of $\boldsymbol{T}_{h}$ and its associated eigenspace is $\left\{\boldsymbol{\tau}_{h} \in \mathcal{W}_{h}\right.$ : $\left.\tau_{h}^{\mathrm{D}}=0\right\}$.

Proof. Since $\mathcal{W}_{h}=\mathcal{K}_{h} \oplus \boldsymbol{P}_{h}\left(\mathcal{W}_{h}\right),\left.\boldsymbol{T}_{h}\right|_{\mathcal{K}_{h}}: \mathcal{K}_{h} \longrightarrow \mathcal{K}_{h}$ is the identity and $\boldsymbol{T}_{h}\left(\boldsymbol{P}_{h}\left(\mathcal{W}_{h}\right)\right) \subset \boldsymbol{P}_{h}\left(\mathcal{W}_{h}\right)$ (cf. Lemma 4.3), the theorem follows from Lemmas 4.1 and the definition of $\boldsymbol{T}_{h}$.

Remark 4.1. The eigenspace of $\boldsymbol{T}_{h}$ corresponding to $\mu_{h}=0$ can be characterized by

$$
\left\{\boldsymbol{\tau}_{h} \in \mathcal{W}_{h}: \boldsymbol{\tau}_{h}^{\mathrm{D}}=0\right\}=\left\{q_{h} \boldsymbol{I}: q_{h} \in V_{h}\right\}
$$

where

$$
V_{h}:=\left\{q_{h} \in \mathrm{H}^{1}(\Omega):\left.q_{h}\right|_{T} \in \mathcal{P}_{1}(T) \forall T \in \mathcal{T}_{h}(\Omega) \text {, and } q=0 \text { on } \Sigma\right\} .
$$

This follows from the same arguments used in the proof of Theorem 3.1(iii) and the fact that for all $q_{h} \in V_{h}, q_{h} \boldsymbol{I} \in \mathcal{W}_{h}$. We notice that this characterization does not necessarily hold true for other conforming discretization of $\mathrm{H}($ div; $\Omega)$ like the Raviart-Thomas mixed finite element.

\section{Spectral approximation}

To prove that $\boldsymbol{T}_{h}$ provides a correct spectral approximation of $\boldsymbol{T}$, we will resort to the theory developed in [8] for noncompact operators. To this end, we first introduce some notations. For any linear operator $S: \mathcal{W} \longrightarrow \mathcal{W}$, we define the norm

$$
\|\boldsymbol{S}\|_{h}:=\sup _{\tau_{h} \in \mathcal{W}_{h}} \frac{\left\|\boldsymbol{S} \tau_{h}\right\|_{\mathrm{H}(\operatorname{div} ; \Omega)}}{\left\|\boldsymbol{\tau}_{h}\right\|_{\mathrm{H}(\operatorname{div} ; \Omega)}} .
$$

Given $\tau \in \mathcal{W}$ and two closed subspaces $\mathcal{Y}$ and $\mathcal{Z}$ of $\mathcal{W}$, we set

$$
\delta(\boldsymbol{\tau}, \mathcal{Y}):=\inf _{\boldsymbol{y} \in \mathcal{Y}}\|\boldsymbol{\tau}-\boldsymbol{y}\|_{\mathrm{H}(\operatorname{div} ; \Omega)}, \quad \delta(\mathcal{Y}, \mathcal{Z}):=\sup _{\boldsymbol{y} \in \mathcal{Y}:\|\boldsymbol{y}\|_{\mathrm{H}(\mathrm{div} ; \Omega)}=1} \delta(\boldsymbol{y}, \mathcal{Z})
$$

and

$$
\widehat{\delta}(\mathcal{Y}, \mathcal{Z}):=\max \{\delta(\mathcal{Y}, \mathcal{Z}), \delta(\mathcal{Z}, \mathcal{Y})\} .
$$

The number $\widehat{\delta}(\mathcal{Y}, \mathcal{Z})$ is usually called the gap between subspaces $\mathcal{Y}$ and $\mathcal{Z}$.

The theory from [8] guaranties a good approximation of the spectrum of $\boldsymbol{T}$ if the following two properties are satisfied:

- (P1): $\left\|\boldsymbol{T}-\boldsymbol{T}_{h}\right\|_{h} \rightarrow 0, \quad$ as $h \rightarrow 0$,

- (P2): $\forall \boldsymbol{\tau} \in \mathcal{W}, \quad \lim _{h \rightarrow 0} \delta\left(\boldsymbol{\tau}, \mathcal{W}_{h}\right)=0$. 
Property (P2) follows immediately from the approximation property of the finite element space (4.4) and the density of smooth functions in $\mathcal{W}$. Hence, it only remains to show that property $(\mathrm{P} 1)$ holds true.

Lemma 5.1. There exists $C>0$, independent of $h$, such that

$$
\left\|\boldsymbol{T}-\boldsymbol{T}_{h}\right\|_{h} \leq C h^{s} .
$$

Proof. Given $\boldsymbol{\sigma}_{h} \in \mathcal{W}_{h}$, we have that

$$
\begin{aligned}
\left(\boldsymbol{T}-\boldsymbol{T}_{h}\right)\left(\boldsymbol{\sigma}_{h}\right) & =\left(\boldsymbol{T}-\boldsymbol{T}_{h}\right)\left(\boldsymbol{P}_{h} \boldsymbol{\sigma}_{h}\right)+\left(\boldsymbol{T}-\boldsymbol{T}_{h}\right)\left(\left(\boldsymbol{I}-\boldsymbol{P}_{h}\right)\left(\boldsymbol{\sigma}_{h}\right)\right) \\
& =\left(\boldsymbol{T}-\boldsymbol{T}_{h}\right)\left(\boldsymbol{P}_{h} \boldsymbol{\sigma}_{h}\right),
\end{aligned}
$$

where the last identity is justified by the fact that $\left(\boldsymbol{I}-\boldsymbol{P}_{h}\right)$ is a projector onto $\operatorname{Ker}\left(\boldsymbol{P}_{h}\right)=\mathcal{K}_{h} \subset \mathcal{K}$. We recall that the restriction of both $\boldsymbol{T}$ and $\boldsymbol{T}_{h}$ to this subspace reduces to the identity. Let us consider now the splitting

$$
\left(\boldsymbol{T}-\boldsymbol{T}_{h}\right)\left(\boldsymbol{P}_{h} \boldsymbol{\sigma}_{h}\right)=\underbrace{\left(\boldsymbol{T}-\widetilde{\boldsymbol{T}}_{h}\right)\left(\left(\boldsymbol{P}_{h}-\boldsymbol{P}\right)\left(\boldsymbol{\sigma}_{h}\right)\right)}_{E_{1}}+\underbrace{\left(\boldsymbol{T}-\widetilde{\boldsymbol{T}}_{h}\right)\left(\boldsymbol{P} \boldsymbol{\sigma}_{h}\right)}_{E_{2}} .
$$

For the first term we use Lemma 4.2 (ii) to obtain the estimate

$$
\left\|E_{1}\right\|_{\mathrm{H}(\mathbf{d i v} ; \Omega)} \leq\left(\|\boldsymbol{T}\|+\left\|\widetilde{\boldsymbol{T}}_{h}\right\|\right)\left\|\left(\boldsymbol{P}_{h}-\boldsymbol{P}\right)\left(\boldsymbol{\sigma}_{h}\right)\right\|_{\mathrm{H}(\mathbf{d i v} ; \Omega)} \leq C_{1} h^{s}\left\|\boldsymbol{\sigma}_{h}\right\|_{\mathrm{H}(\mathbf{d i v} ; \Omega)} .
$$

By virtue of Céa estimate (4.5), the second one is bounded as follows

$$
\left\|E_{2}\right\|_{\mathrm{H}(\operatorname{div} ; \Omega)} \leq C_{2} \inf _{\boldsymbol{\tau}_{h} \in \mathcal{W}_{h}}\left\|\boldsymbol{T}\left(\boldsymbol{P} \boldsymbol{\sigma}_{h}\right)-\boldsymbol{\tau}_{h}\right\|_{\mathrm{H}(\operatorname{div} ; \Omega)} .
$$

Now, according to Proposition 3.1, if we denote $\boldsymbol{\sigma}^{*}=\boldsymbol{T}\left(\boldsymbol{P} \boldsymbol{\sigma}_{h}\right)$, then $\boldsymbol{\sigma}^{*} \in \mathrm{H}^{s}(\Omega)^{n \times n}$, $\operatorname{div} \boldsymbol{\sigma}^{*} \in \mathrm{H}^{1}(\Omega)^{n}$ and

$$
\left\|\boldsymbol{\sigma}^{*}\right\|_{s, \Omega}+\left\|\operatorname{div} \boldsymbol{\sigma}^{*}\right\|_{1, \Omega} \leq C_{3}\left\|\boldsymbol{P} \boldsymbol{\sigma}_{h}\right\|_{\mathrm{H}(\operatorname{div} ; \Omega)} \leq C_{4}\left\|\boldsymbol{\sigma}_{h}\right\|_{\mathrm{H}(\operatorname{div} ; \Omega)} .
$$

We deduce from the two last estimates and the approximation property (4.4) that

$$
\left\|E_{2}\right\|_{\mathrm{H}(\operatorname{div} ; \Omega)} \leq C_{2} \inf _{\boldsymbol{\tau}_{h} \in \mathcal{W}_{h}}\left\|\boldsymbol{\sigma}^{*}-\boldsymbol{\tau}_{h}\right\|_{\mathrm{H}(\operatorname{div} ; \Omega)} \leq C_{5} h^{s}\left\|\boldsymbol{\sigma}_{h}\right\|_{\mathrm{H}(\operatorname{div} ; \Omega)} .
$$

Summing up, we have shown that

$$
\left\|\left(\boldsymbol{T}-\boldsymbol{T}_{h}\right)\left(\boldsymbol{\sigma}_{h}\right)\right\|_{\mathrm{H}(\mathbf{d i v} ; \Omega)} \leq C_{6} h^{s}\left\|\boldsymbol{\sigma}_{h}\right\|_{\mathrm{H}(\operatorname{div} ; \Omega)} \quad \boldsymbol{\sigma}_{h} \in \mathcal{W}_{h}
$$

with $C_{6}$ independent of $h$ which proves the lemma. $1]$.

The following result is a consequence of properties (P1) and (P2), see $[8$, Theorem

Theorem 5.1. Let $F \subset \mathbb{C}$ be an open set containing $\operatorname{sp}(\boldsymbol{T})$. Then, there exist $h_{0}>0$ such that $\operatorname{sp}\left(\boldsymbol{T}_{h}\right) \subset F$, for all $h<h_{0}$.

This theorem means that our Galerkin scheme does not introduce spurious modes with eigenvalues interspersed among the positive eigenvalues of Problem 1. Indeed, assume that $\mu \in(0,1)$ is an isolated eigenvalue of $\boldsymbol{T}$ with finite multiplicity $m$ and that $\mathcal{C}$ is an open circle in the complex plane centered at $\mu$ with boundary $\gamma$, such that $\mu$ is the only eigenvalue of $\boldsymbol{T}$ lying in $\mathcal{C}$ and $\gamma \cap \operatorname{sp}(\boldsymbol{T})=\emptyset$. Then, according to [8, Section 2], for $h$ small enough, there exist $m$ eigenvalues $\mu_{h 1}, \ldots, \mu_{h m}$ of $\boldsymbol{T}_{h}$ (repeated accordingly to their respective multiplicities) converging to $\mu$ as $h \rightarrow 0$.

The next step in our analysis is to to apply the results from [9] to obtain error estimates. To this aim, we consider the eigenspace $\mathcal{E}$ of $\boldsymbol{T}$ corresponding to $\mu$ and the invariant subspace $\mathcal{E}_{h}$ of $\boldsymbol{T}_{h}$ spanned by the eigenspaces of $\boldsymbol{T}_{h}$ corresponding 
to $\mu_{h 1}, \ldots, \mu_{h m}$. Since $\boldsymbol{T}$ and $\boldsymbol{T}_{h}$ are self-adjoint with respect o $a(\cdot, \cdot)$, we have the following results from [9].

Theorem 5.2. There exists a constant $C>0$, independent of $h$ such that

$$
\widehat{\delta}\left(\mathcal{E}_{h}, \mathcal{E}\right) \leq C \delta\left(\mathcal{E}, \mathcal{W}_{h}\right) .
$$

Proof. The result follows from arguments similar to those used in the proof of $[9$, Theorem 1].

We recall that $\mu \in(0,1)$ is an eigenvalue of $\boldsymbol{T}$ with multiplicity $m$ if and only $\lambda:=(1 / \mu)-1$ is an eigenvalue of Problem 1 with the same multiplicity and the corresponding eigenfunctions coincide. Analogously, $\mu_{h i}, i=1, \ldots, m$, are the eigenvalues of $\boldsymbol{T}_{h}$ (repeated accordingly to their respective multiplicities) converging to $\mu$ as $h$ approaches zero if and only if $\lambda_{h i}:=\left(1 / \mu_{h i}\right)-1$ are the eigenvalues of Problem 3 converging to $\lambda$ and the corresponding eigenfunctions also coincide. Thus, the theorem above provides an error estimate for the eigenfunctions of Problem 1. Finally, we have the following result that provides an error estimate for the eigenvalues.

Theorem 5.3. There exist constant $C>0$, independent of $h$ such that for all $h$ small enough

$$
\left|\lambda-\lambda_{h i}\right| \leq C \delta\left(\mathcal{E}, \mathcal{W}_{h}\right)^{2}, \quad i=1, \ldots, m .
$$

Proof. A proof of this result is given in [9, Theorem 3], but for the sake of completeness, we include here a simpler demonstration that is well adapted for our case.

Let $\sigma_{h i}$ be an eigenfunction of Problem 3 corresponding to $\lambda_{h i}$, normalized so that $\left\|\boldsymbol{\sigma}_{h i}\right\|_{\mathrm{H}(\operatorname{div} ; \Omega)}=1$. According to Theorem 5.2, $\delta\left(\boldsymbol{\sigma}_{h i}, \mathcal{E}\right) \leq C \delta\left(\mathcal{E}, \mathcal{W}_{h}\right)$. It follows that there exists an eigenfunction $\sigma \in \mathcal{E}$ of Problem 1 corresponding to $\lambda$ such that

$$
\left\|\boldsymbol{\sigma}-\boldsymbol{\sigma}_{h i}\right\|_{\mathrm{H}(\mathrm{div} ; \Omega)} \leq C \delta\left(\mathcal{E}, \mathcal{W}_{h}\right)
$$

The key tool in the prove the theorem is the identity (see, for instance, $[1$, Lemma 9.1])

$$
\left(\lambda_{h i}-\lambda\right) b\left(\boldsymbol{\sigma}_{h i}, \boldsymbol{\sigma}_{h i}\right)=a\left(\boldsymbol{\sigma}-\boldsymbol{\sigma}_{h i}, \boldsymbol{\sigma}-\boldsymbol{\sigma}_{h i}\right)-(\lambda+1) b\left(\boldsymbol{\sigma}-\boldsymbol{\sigma}_{h i}, \boldsymbol{\sigma}-\boldsymbol{\sigma}_{h i}\right) .
$$

It is obtained by straightforward calculations after noticing that, by virtue of Problems 1 and 3 ,

$$
\begin{aligned}
& a(\boldsymbol{\sigma}, \boldsymbol{\tau})=(\lambda+1) b(\boldsymbol{\sigma}, \boldsymbol{\tau}) \quad \forall \boldsymbol{\tau} \in \mathcal{W}, \\
& a\left(\boldsymbol{\sigma}_{h i}, \boldsymbol{\tau}_{h}\right)=\left(\lambda_{h i}+1\right) b\left(\boldsymbol{\sigma}_{h i}, \boldsymbol{\tau}_{h}\right) \quad \forall \boldsymbol{\tau}_{h} \in \mathcal{W}_{h} .
\end{aligned}
$$

Lemma 2.1 and the fact that $\lambda_{h i} \rightarrow \lambda$ as $h$ goes to zero yield

$$
b\left(\boldsymbol{\sigma}_{h i}, \boldsymbol{\sigma}_{h i}\right)=\frac{a\left(\boldsymbol{\sigma}_{h i}, \boldsymbol{\sigma}_{h}\right)}{\lambda_{h i}+1} \geq \frac{\alpha\left\|\boldsymbol{\sigma}_{h i}\right\|_{\mathrm{H}(\mathbf{d i v} ; \Omega)}^{2}}{\lambda_{h i}+1} \geq \tilde{C} .
$$

Using the last estimate to bound the left hand side of (5.2) from below and the continuity of $a$ and $b$ and (5.1) to bound the right hand side from above we deduce that

which proves the theorem.

$$
\left|\lambda-\lambda_{h i}\right| \leq C \delta\left(\mathcal{E}, \mathcal{W}_{h}\right)^{2}, \quad i=1, \ldots, m,
$$


The two theorems above yield error estimates depending on $\delta\left(\mathcal{E}, \mathcal{W}_{h}\right)$. The order of convergence will then depend on the regularity of the eigenfunctions. Since we already proved additional smoothness of these eigenfunctions (see (4.4) and Corollary 3.1), we can assert that

$$
\left\|\boldsymbol{\sigma}-\boldsymbol{\Pi}_{h} \boldsymbol{\sigma}\right\|_{\mathrm{H}(\operatorname{div} ; \Omega)} \leq C h^{s}\|\boldsymbol{\sigma}\|_{\mathrm{H}^{s}(\operatorname{div} ; \Omega)} \leq C h^{s}\|\boldsymbol{\sigma}\|_{\mathrm{H}(\operatorname{div} ; \Omega)} \quad \forall \boldsymbol{\sigma} \in \mathcal{E},
$$

which proves that

$$
\delta\left(\mathcal{E}, \mathcal{W}_{h}\right) \leq C h^{s}
$$

\section{Numerical Results}

We present in this section results from numerical tests carried out with the method proposed in Section 4 to confirm the theoretical results proved above. The numerical method was implemented in a MATLAB code. We used uniform meshes as shown in Figure 1. The refinement parameter $N$ used to label each mesh is the number of elements on each edge. In all the tables below the results are obtained by using four successive meshes with an increasing level of refinement. In each case, we list the lowest eigenvalues $\lambda_{h i}$ of Problem 3 and give an estimate of the order of convergence of the Galerkin scheme. We also report a more accurate extrapolated approximation of the eigenvalues obtained by a least-squares fitting.

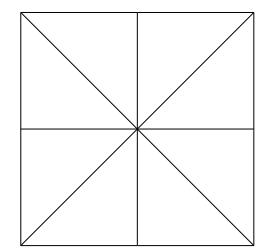

$N=2$

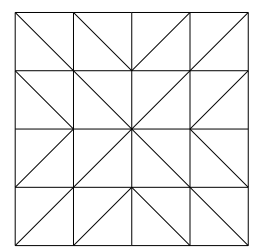

$N=4$

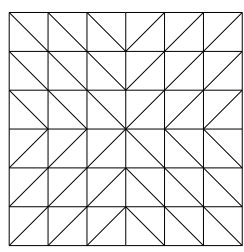

$N=6$

FiguRE 1. Uniform meshes.

6.1. Test 1: In this numerical test we take $\Omega:=(-1,1) \times(-1,1)$ and consider a non-slip boundary conditions on the whole $\partial \Omega$. In Table 1 , we compare our results with those obtained in [17] for a velocity-pressure formulation of the Stokes system and a Galerkin method based on the MINI-element.

TABLE 1. Lowest eigenvalues $\lambda_{h i}, i=1, \ldots, 6$ of the Stokes problem computed with the method analyzed in this paper.

\begin{tabular}{cccccccc}
\hline & $N=10$ & $N=20$ & $N=30$ & $N=40$ & Order & Extrapolated & {$[17]$} \\
\hline$\lambda_{h 1}$ & 13.4657 & 13.1823 & 13.1290 & 13.1103 & 1.98 & 13.0860 & 13.086 \\
$\lambda_{h 2}=\lambda_{h 3}$ & 24.2868 & 23.3472 & 23.1718 & 23.1103 & 1.99 & 23.0308 & 23.031 \\
$\lambda_{h 4}$ & 34.2444 & 32.6220 & 32.3075 & 32.1963 & 1.93 & 32.0443 & 32.053 \\
$\lambda_{h 5}$ & 41.4711 & 39.2828 & 38.8666 & 38.7201 & 1.96 & 38.5252 & 38.532 \\
$\lambda_{h 6}$ & 45.9681 & 42.8124 & 42.2263 & 42.0211 & 2.00 & 41.7588 & 41.759 \\
\hline
\end{tabular}

It is clear that the results obtained by the two methods agree and that the eigenvalue approximation order is quadratic. We also point out that the symmetry 
of the mesh permits to preserve the double multiplicity of the second eigenvalue at the discrete level.

6.2. Test 2: In this numerical test we compare our method with a finite element method analyzed in [20] to solved the plate buckling problem. The computational domain is given this time by the unit square $\Omega:=(0,1) \times(0,1)$. As we already mentioned in the introduction, the Stokes eigenvalue problem with homogeneous boundary conditions is equivalent to the plate buckling problem (see [7, 19]): Find $\lambda \in \mathbb{R}, 0 \neq w \in \mathrm{H}_{0}^{2}(\Omega)$, such that

$$
\left\{\begin{aligned}
\Delta^{2} w & =-\lambda \Delta w & & \text { in } \Omega, \\
w & =0 & & \text { on } \partial \Omega, \\
\nabla w \cdot \nu & =0 & & \text { on } \partial \Omega
\end{aligned}\right.
$$

where the stream function $w$ is related to the velocity by $\boldsymbol{u}=\operatorname{curl} w=\left(\partial_{2} w,-\partial_{1} w\right)$.

In Table 2, we list the lowest eigenvalues of problem (6.1) computed by using the finite element method analyzed in [20]. These results are to be compared with those of Table 3 where the eigenvalues are obtained by solving Problem 3. It is clear that our method computes the buckling intensity of a uniformly compressed clamped plate with a quasi-optimal order of convergence.

TABLE 2. Lowest eigenvalues $\lambda_{h i}, i=1, \ldots, 6$ of problem (6.1) computed with the method from [20].

\begin{tabular}{ccccccc}
\hline & $N=10$ & $N=20$ & $N=30$ & $N=40$ & Order & Extrapolated \\
\hline$\lambda_{h 1}$ & 52.3690 & 52.3489 & 52.3464 & 52.3456 & 2.61 & 52.3450 \\
$\lambda_{h 2}=\lambda_{h 3}$ & 92.4666 & 92.2058 & 92.1601 & 92.1444 & 2.09 & 92.1257 \\
$\lambda_{h 4}$ & 128.8540 & 128.3586 & 128.2745 & 128.2458 & 2.14 & 128.2132 \\
$\lambda_{h 5}$ & 153.7617 & 154.0403 & 154.0877 & 154.1042 & 2.12 & 154.1232 \\
$\lambda_{h 6}$ & 169.3163 & 167.6182 & 167.2925 & 167.1776 & 1.95 & 167.0241 \\
\hline
\end{tabular}

TABLE 3. Lowest eigenvalues $\lambda_{h i}, i=1, \ldots, 6$ of the Stokes problem computed with the method analyzed in this paper.

\begin{tabular}{ccccccc}
\hline & $N=10$ & $N=20$ & $N=30$ & $N=40$ & Order & Extrapolated \\
\hline$\lambda_{h 1}$ & 52.7294 & 52.4412 & 52.3876 & 52.3689 & 1.99 & 52.3444 \\
$\lambda_{h 2}=\lambda_{h 3}$ & 93.3889 & 92.4412 & 92.2653 & 92.2036 & 2.00 & 92.1249 \\
$\lambda_{h 4}$ & 130.4880 & 128.7851 & 128.4659 & 128.3538 & 1.98 & 128.2070 \\
$\lambda_{h 5}$ & 157.1313 & 154.8806 & 154.4614 & 154.3145 & 1.99 & 154.1236 \\
$\lambda_{h 6}$ & 171.2498 & 168.0844 & 167.4982 & 167.2930 & 2.00 & 167.0293 \\
\hline
\end{tabular}

6.3. Test 3: In this numerical test we will consider our method applied to the Stokes eigenvalue problem with mixed boundary conditions. The computational domain is again the unit square $\Omega:=(0,1) \times(0,1)$ and $\Gamma:=(0,1) \times\{0\}$. We can see from the results reported in Table 4 that, in this case, the order of convergence is also quadratic. 
TABLE 4. Lowest eigenvalues $\lambda_{h i}, i=1, \ldots, 6$ of the Stokes problem computed with the method analyzed in this paper.

\begin{tabular}{ccccccc}
\hline & $N=10$ & $N=20$ & $N=30$ & $N=40$ & Order & Extrapolated \\
\hline$\lambda_{h 1}$ & 2.4708 & 2.4682 & 2.4678 & 2.4676 & 2.00 & 2.4674 \\
$\lambda_{h 2}$ & 6.2946 & 6.2835 & 6.2813 & 6.2805 & 1.85 & 6.2793 \\
$\lambda_{h 3}$ & 15.3288 & 15.2402 & 15.2232 & 15.2171 & 1.94 & 15.2090 \\
$\lambda_{h 4}$ & 22.4812 & 22.2751 & 22.2371 & 22.2237 & 2.00 & 22.2065 \\
$\lambda_{h 5}$ & 27.3583 & 27.0518 & 26.9945 & 26.9744 & 1.98 & 26.9479 \\
$\lambda_{h 6}$ & 44.2217 & 43.4121 & 43.2619 & 43.2093 & 2.00 & 43.1419 \\
\hline
\end{tabular}

Finally, we show in Figures 2 and 3 the eigenfunctions corresponding to the lowest four eigenvalues.
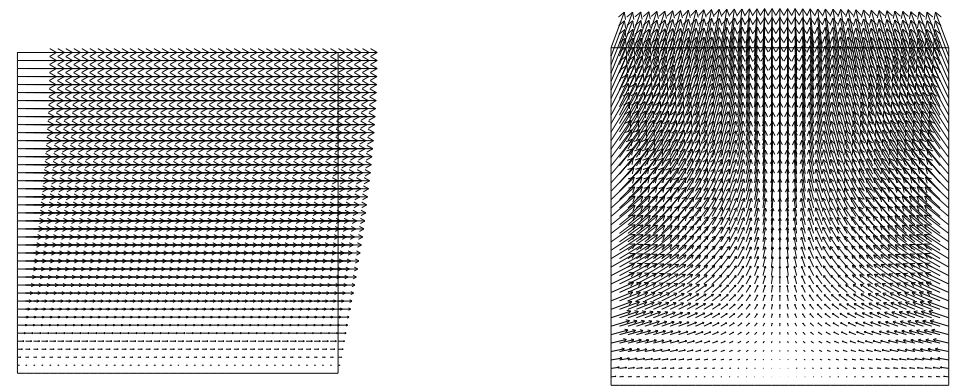

Figure 2. Eigenfunctions associated to eigenvalues $\lambda_{h 1}$ (left) and $\lambda_{h 2}$ (right).
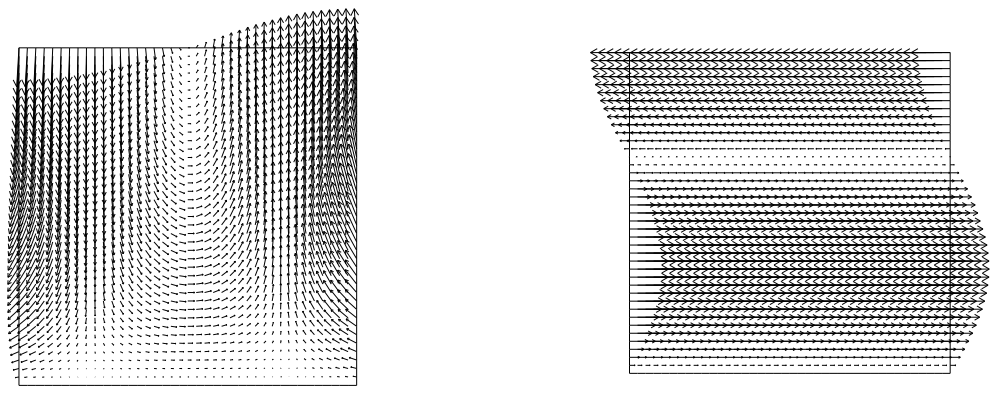

Figure 3. Eigenfunctions associated to eigenvalues $\lambda_{h 3}$ (left) and $\lambda_{h 4}$ (right). 


\section{REFERENCES}

[1] I. BABušKa And J. Osborn, Eigenvalue Problems, in Handbook of Numerical Analysis, Vol. II, P. G. Ciarlet and J. L. Lions, eds., North-Holland, Amsterdam, 1991, pp. 641-787.

[2] A. Bermúdez, R. Durán, M.A. Muschietti, R. Rodríguez, and J. Solomin, Finite element vibration analysis of fluid-solid systems without spurious modes, SIAM J. Numer. Anal., 32 (1995) 1280-1295.

[3] D. Boffi, Finite element approximation of eigenvalue problems. Acta Numerica, vol. 19 (2010) 1-120.

[4] D. Boffi, F. Brezzi and L. Gastaldi, On the convergence of eigenvalues for mixed formulations, Ann. Scuola Norm. Sup. Pisa Cl. Sci. (4) 25(1-2), (1997) 131-154.

[5] F. Brezzi And M. Fortin, Mixed and Hybrid Finite Element Methods, Springer Verlag, New York, 1991.

[6] Z. Cai, T. Charles, P.S. Vassilevski and C. Chunbo, Mixed finite element methods for incompressible flow: stationary Stokes equations. Numer. Methods Partial Differential Equations, 26 (2010) 957-978.

[7] W. Chen, And Q. Lin, Approximation of an eigenvalue problem associated with the Stokes problem by the stream function-vorticity-pressure method. Appl. Math., 51(1) (2006) 73-88.

[8] J. Descloux, N. Nassif, and J. Rappaz, On spectral approximation. Part 1: The problem of convergence, RAIRO Anal. Numér., 12(2) (1978) 97-112.

[9] J. Descloux, N. Nassif, And J. Rappaz, On spectral approximation. Part 2: Error estimates for the Galerkin method, RAIRO Anal. Numér., 12(2) (1978) 113-119.

[10] E.B. Fabes, C.E. Kenig, and G.C. Verchota, The Dirichlet problem for the Stokes system on Lipschitz domains, Duke Math. J., 57(3) (1988) 769-793.

[11] G.N. Gatica, Analysis of a new augmented mixed finite element method for linear elasticity allowing $\mathbb{R T}_{0}-\mathbb{P}_{1}-\mathbb{P}_{0}$ approximations, M2AN Math. Model. Numer. Anal., 40(1) (2006) $1-28$.

[12] G.N. Gatica, A. Márquez, And M.A. SÁnchez, Analysis of a velocity-pressure-pseudostress formulation for the stationary Stokes equations, Comput. Methods Appl. Mech. Engrg., 199(17-20) (2010) 1064-1079.

[13] G.N. Gatica, A. Márquez, and M.A. SÁnchez, Pseudostress-based mixed finite element methods for the Stokes problem in $\mathbb{R}^{n}$ with Dirichlet boundary conditions. I: A priori error analysis, Commun. Comput. Phys., 12(1) (2012) 109-134.

[14] V. Girault, and P. A. Raviart, Finite element methods for Navier-Stokes equations. Theory and algorithms, Springer-Verlag, Berlin, 1986.

[15] R. Hiptmair, Finite elements in computational electromagnetism. Acta Numerica, vol. 11 (2002) 237-339.

[16] E. Leriche, AND G. LABrosse, Stokes eigenmodes in square domain and the stream functionvorticity correlation. J. Comput. Phys., 200(2) (2004) 489-511.

[17] C. Lovadina, M. Lyly and R. Stenberg, A posteriori estimates for the Stokes eigenvalue problem. Numer. Methods Partial Differential Equations, vol. 25 (2009) 244-257.

[18] S. Meddahi, D, Mora, and R. RodríGuez, Finite element spectral analysis for the mixed formulation of the elasticity equations. SIAM J. Numer. Anal., 51(2) (2013) 1041-1063.

[19] B. Mercier, J. Osborn, J. Rappaz, and P.A. Raviart, Eigenvalue approximation by mixed and hybrid methods. Math. Comp. 36 (1981) 427-453.

[20] D. Mora, And R. Rodríguez, A piecewise linear finite element method for the buckling and the vibration problems of thin plates. Math. Comp. 78(268) (2009) 1891-1917.

[21] G. Savaré, Regularity results for elliptic equations in Lipschitz domains, J. Funct. Anal., 152(1) (1998) 176-201. 
Departamento de Matemáticas, Facultad de Ciencias, Universidad de Oviedo, Calvo Sotelo s/n, Oviedo, Spain.

E-mail address: salim@uniovi.es

Departamento de Matemática, Universidad del Bío-Bío, Casilla 5-C, Concepción, Chile and Centro de Investigación en Ingeniería Matemática ( $\mathrm{CI}^{2} \mathrm{MA}$ ), Universidad de Concepción, Concepción, Chile.

E-mail address: dmora@ubiobio.cl

CI²MA, Departamento de Ingeniería Matemática, Universidad de Concepción, Casilla 160-C, Concepción, Chile.

E-mail address: rodolfo@ing-mat.udec.cl 